\title{
Micro Emprendimientos Familiares de Calzado en Tungurahua.
}

\section{Micro Family Enterprises of footwear in Tungurahua.}

Vargas Alex. ${ }^{1}$, Reinoso Glenda. ${ }^{2} \&$ Pico Ibeth. ${ }^{3}$

DOI: : https://doi.org/10.33262/visionariodigital.v1i2.239

\section{Resumen.}

El presente artículo se trata sobre los microemprendimientos de calzado en la Provincia de Tungurahua, donde estos son pequeñas empresas de no máximo de diez personas, por lo general los operarios son los mismos familiares, pero que generan ganancias, fuentes de empleo y el crecimiento del PIB en nuestro país. Estos pequeños emprendimientos u organizaciones nacen en las zonas rurales del país, por la necesidad de salir de problemas económicos que enfrentan las familias ecuatorianas y dar solución al desempleo. También se lo conoce como autoempleo ya que surgen gracias a las propias ideas de los emprendedores. Los microemprendimientos están basados en el objetivo número tres del plan del buen vivir del Ecuador el cual consta de mejorar la calidad de vida de los ecuatorianos. Al igual que su relación con el objetivo número nueve del plan del buen vivir el cual nos dice que nos garantiza un trabajo digno en todas sus formas.

Palabras clave: Microemprendimientos, desempleo, económicos, empleo, calzado.

\begin{abstract}
.
The present article treats itself on the familiar micro employment in the Ecuador, where these are small enterprises of not maximum of ten persons, in general the operatives are the same relatives, but that generate earnings, sources of employment and the growth of the GDP in our country. These small employment or organizations are born in the rural zones of the country, for the need to go out of economic problems that face the Ecuadoran families and to give solution to the unemployment. In addition, it is known as auto employment since they arise thanks to the own ideas of the entrepreneurs. In addition, it is known as self-employment since they arise thanks to the ideas of entrepreneurs. Micro-
\end{abstract}

${ }^{1}$ Universidad Técnica de Ambato, FCADM, Ecuador, alexvargastrilli@ hotmail.com

${ }^{2}$ Universidad Técnica de Ambato, FCADM, Ecuador, glendys_25@yahoo.com ${ }^{3}$ Universidad Técnica de Ambato.FCADM. Ecuador, ibethpicorobles@ @otmail.com 
enterprises are based on objective number three of the plan of the good living of Ecuador, which consists of improving the quality of life of Ecuadorians. As well as its relationship with the target number nine of the plan of the good living which tells us which guarantees us a decent work in all its forms.

Key words: Mike employments, unemployment, economic, employment, families.

\section{Introducción.}

Los Micro emprendimientos se han constituido como un fenómeno que surge especialmente en las zonas rurales del país a partir del deterioro generalizado de las condiciones de ingreso y empleo de los sectores populares, se observa en estas organizaciones una tendencia al surgimiento de generación de empleo e ingresos con un apoyo importante por parte del Gobierno puesto que aporta con un porcentaje importante en el PIB.

Uno de los objetivos de los microemprendimientos es mejorar la calidad de vida de los ecuatorianos, y garantizar un trabajo digno en todas sus formas este está respaldado en el Plan Nacional del Buen Vivir.

No podemos hablar de un impacto masivo de este tipo de micro emprendimientos apenas es una mínima porción del universo de este tipo de actividades, esto nos llevó a realizar una investigación que se refiere a las condiciones funcionales acerca de estos micros emprendimientos.

La manera más frecuente a salir de situaciones de desempleo o falta de actividad laboral es el autoempleo o la idealización de micro emprendimientos, donde los trabajadores informales son aquellos operarios por cuenta propia en los emprendimientos familiares.

Esta situación de poder resolver el problema de la subsistencia en el mercado de trabajo formal hace que estas, estrategias de generación de ingresos formen parte de las estrategas de supervivencia que despliega el grupo domestico para poder garantizar su subsistencia.

No todas las actividades económicas de autoempleo tienen a conformarse micro emprendimientos, es posible que estén implícitos recursos y estrategias de supervivencia diferentes a las de otras actividades informales.

La mayor parte de los microemprendimientos establecidos en las zonas rurales establecen un vínculo con organismos gubernamentales, no gubernamentales, etc.

\section{Metodología.}

El presente artículo se ha desarrollado mediante una investigación participativa en la que se utiliza metodología de observación e investigación para el análisis de la información. El artículo que a continuación se presenta se ha desarrollado tomando en cuenta la cantidad de micro emprendimientos que existe en la ciudad de Ambato como sus diferentes tipos. 
Gran parte de los micro emprendimientos familiares han surgido en base a la necesidad de tener un trabajo ya que en su mayoría la empezar el emprendimiento eran parte de la población inactiva, al utilizar la metodología mencionada anteriormente se observó que los micro emprendimientos en su mayoría están ubicados en la zona rural de algún sector.

Dicha metodología se diseñó específicamente para alcanzar dos propósitos:

a) Recoger información sobre la cantidad, tipos y causas del micro emprendimientos familiares.

b) Analizar este sector productivo del país como son las fuentes de empleo, el aporte al crecimiento del PIB, la participación y apoyo del Gobierno.

Por tratarse de una investigación participativa, es empírica e implica el involucramiento activo de la población con la que se trabaja, además de la utilización de un muestreo intencional, es decir los datos recaudados se seleccionaran según los criterios e información requerida.

\section{Revisión literaria.}

Hace muchos años atrás las microempresas o microemprendimientos, son un tema muy importante en la opinión de las personas. Tanto los microemprendimientos como las pequeñas, medianas y grandes empresas con su triunfante desempeño tienen incidencias en lo que se refiere al desarrollo económico da cada país. Existe una gran unión en la PYMES, ya que posiblemente son conocidas como las más firmes y hacen a un lado a las microempresas especialmente en al momento de hablar de mantenerse en el mercado.

Las pequeñas empresas nacen especialmente en los países latinoamericanos, en nuestro caso en el Ecuador en exclusivo por la necesidad de salir de problemas económicos, la mayoría de estos microemprendimientos son nombrados de tipo informal, es decir una organización que surge espontáneamente y naturalmente entre las personas que ocupan posiciones en la organización formal y a partir de las relaciones que se establecen entre sí como ocupantes de cargos. Pero asi como los que recién nacen, a los que ya llevan un tiempo en el mercado son declarados como microemprendimientos formales, las cuales están basadas en la división del trabajo, las pequeñas organizaciones son considerados como mecanismos para salir de la pobreza, la cual es la falta de recursos materiales, o falta del desarrollo de las capacidades de una persona.

Es por eso que es lógico realizar una revisión de este tema basándonos en la literatura, los factores importantes que llevan al éxito a un micro emprendimiento, sabiendo que el éxito es la subsistencia, la superación y las ganancias que se consiguen con estas organizaciones. Para crear una buena idea o ser un excelente micro emprendedor, tiene que haber pasado por una buena educación tanto primaria, secundaria y universitaria, y capacitaciones para asi alcanzar el éxito del micro emprendimiento, además se puede obtener un buen acceso a un capital financiero para tener alternativas de financiamiento, para asi hacer posible que se generen nuevas y mejores actividades productivas y la innovación del producto. 
Para que estos proyectos se mantengan en el mercado los micros emprendedores deben destacarse en la formación obtenida, y la continua capacitación y tener un buen financiamiento. Se necesita tener en cuenta los factores del éxito de una micro empresa, una de ellas es el tamaño del micro emprendimiento, otro es el nivel de endeudamiento que tiene esta mini organización la cual debe ser una deuda que se pueda controlar y no salirse de las manos del propietario, además de una buena infraestructura, contar con tecnología de punta y una excelente comunicación entre empleador y operarios en nuestro caso entre la familia que conforman las pequeñas empresas.

Además, tenemos el entorno en el que se lleva a cabo el micro emprendimiento es decir que esto también tiene una gran influencia y tiene que ver mucho con el éxito del micro emprendimiento, donde se destaca el apoyo económico que recibe por parte del gobierno, no solo económico sino en capacitaciones para el desarrollo de la misma.

Para concluir con el tema debemos determinar que el éxito o el fracaso de los micro emprendimientos se basa en si es más productiva que las otras, la cual se va agrandando si se hace las cosas o actores mencionadas anteriormente, también es tener a un excelente emprendedor para que el proyecto que se está llevando a cabo pueda surgir y asi dar estabilidad económica a la familia emprendedora.

En si este proyecto tiene como finalidad demostrar el índice de pequeñas empresas de calzado que existen en la provincia de Tungurahua, ya que es una de las potencias del ecuador en el procesamiento de cuero, gracias a esto varios microempresarios se ven beneficiados ya que cuentan con el requisito de la materia Price cerca de sus negocios y a un buen precio, además las grandes procesadoras de cuero tienen beneficios para que los pequeños emprendedores obtengan grandes beneficios para la adquisición de su materia prima y no tengan que ir a buscar en otro sector.

Varios reportajes de diarios como el UNIVERSO han catalogado que Tungurahua es el principal promotor de cuero en el país, contando tanto con pequeñas empresas que quieren superarse y seguir creciendo en el mercado como grandes empresas que ya incursionan en este mercado durante varios años como por ejemplo calzado VENUS, una de las marcas preferidas en el mercado, y como ejemplo a seguir de las otras empresas.

Se dice que Ecuador en uno de los países que utilizan los microemprendimientos como una salida de la pobreza la cual nos dice que ayuda tanto al ciudadano que las crea como al gobierno ya que al crecer esta microempresa el estado se ve beneficiado con sus ganancias y asi poder aportar a él. En cifras podemos hablar que en el Ecuador el $90 \%$ de los negocios son microempresas y además son familiares.'

\section{Plan Nacional del Buen Vivir}

\section{Objetivo 2.}

Auspiciar la igualdad, la cohesión, la inclusión y la equidad social y territorial en la diversidad. 
El reconocimiento igualitario de los derechos de todos los individuos implica la consolidación de políticas de igualdad que eviten la exclusión y fomenten la convivencia social y política. El desafío es avanzar hacia la igualdad plena en la diversidad, sin exclusión, para lograr una vida digna, con acceso a salud, educación, protección social, atención especializada y protección especial.

\section{Objetivo 3.}

\section{Mejorar la calidad de vida de la población.}

Mejorar la calidad de vida de la población es reto amplio que demanda la consolidación de los logros alcanzados en los últimos seis años y medio, mediante el fortalecimiento de políticas intersectoriales y la consolidación del Sistema Nacional de Inclusión y Equidad Social.

\section{Objetivo 8.}

Consolidar el sistema económico social y solidario, de forma sostenible.

El sistema económico mundial requiere renovar su concepción, priorizando la igualdad en las relaciones de poder, tanto entre países como al interior de ellos. De igual manera, dando prioridad a la (re)distribución y al ser humano, sobre el crecimiento económico y el capital (Senplades, 2009). Esta nueva concepción permitirá concretar aspectos como la inclusión económica y social de millones de personas, la transformación del modo de producción de los países del Sur, el fortalecimiento de las finanzas públicas, la regulación del sistema económico, y la justicia e igualdad en las condiciones laborales.

\section{Objetivo 9.}

Garantizar el trabajo digno en todas sus formas.

Los principios y orientaciones para el Socialismo del Buen Vivir reconocen que la supremacía del trabajo humano sobre el capital es incuestionable.

De esta manera, se establece que el trabajo no puede ser concebido como un factor más de producción, sino como un elemento mismo del Buen Vivir y como base para el despliegue de los talentos de las personas.

\section{Objetivo 10.}

Impulsar la transformación de la matriz productiva.

Los desafíos actuales deben orientar la conformación de nuevas industrias y la promoción de nuevos sectores con alta productividad, competitivos, sostenibles, sustentables y 
diversos, con visión territorial y de inclusión económica en los encadenamientos que generen. Se debe impulsar la gestión de recursos financieros y no financieros, profundizar la inversión pública como generadora de condiciones para la competitividad sistémica, impulsar la contratación pública y promover la inversión privada.

\section{Objetivo 11.}

Asegurar la soberanía y de los sectores estratégicos para la transformación industrial y tecnológica.

El Ecuador tiene una oportunidad histórica para ejercer soberanamente la gestión económica, industrial y científica, de sus sectores estratégicos. Esto permitirá generar riqueza y elevar en forma general el nivel de vida de nuestra población. Para el Gobierno de la Revolución Ciudadana, convertir la gestión de los sectores estratégicos en la punta de lanza de la transformación tecnológica e industrial del país, constituye un elemento central de ruptura con el pasado.

\section{Estado del arte}

(Bellingi \& Sergio, 2013) "Una de las nuevas formas que asume la política social es la promoción de micro emprendimientos (ME) productivos, entre cuyos objetivos se destaca que los desocupados obtengan ingresos provenientes de su trabajo. Durante largo tiempo se consideró a la actividad microempresaria sólo como un refugio, al que acudían los desempleados o la población más pobre que no tiene acceso al mercado de trabajo formal. Especialmente entre los pobres estructurales, la promoción de tareas productivas destinadas al autoconsumo o al intercambio informal surgía como una alternativa frente a otras políticas puramente asistencialistas. Estos emprendimientos productivos se basan en las propias capacidades de trabajo de los desocupados y requieren poco capital inicial. Debido a esto la gran mayoría genera bajos ingresos, al punto de no permitir salir del sector informal al no poder hacer frente al pago de impuestos u otras normativas legales."

(Blaser, 2003) "El mercado ofrece desafíos a los micro emprendimientos productivos, cuyos integrantes (los emprendedores) responden proveyendo de bienes y servicios, siempre que han estudiado correctamente ese mercado. Además las diferentes organizaciones de la comunidad requieren habilidades de los emprendedores que, a su vez, pueden dar respuesta a las necesidades constituyendo redes sociales que los impulsen y dinamicen.

Sin capital (capital circulante, es decir, dinero, es bastante difícil gestar una empresa. Es necesario primero constituir un micro emprendimiento que permita a través de la relación con otros y de la integración en el contexto social, cubrir las necesidades, incorporar un ingreso a la familia o amplia $\mathrm{r}$ los ingresos y acumular capital. 
Los micros emprendimientos constituyen una organización que generalmente tiene su origen en la cercanía residencial y cuyos pilares son:

- El trabajo en equipo, o trabajo colectivo.

- El compromiso y la responsabilidad de las tareas por parte de los integrantes del equipo.

- La aceptación de un líder en la conducción.

- Una capacitación constante y la adaptación a los cambios económicos y sociales permanentes del mundo actual."

(Glas, 2014) "Este es el momento de cambiar la Matriz Productiva y lo haremos solo con ustedes: los emprendedores", expresó el segundo Mandatario, Jorge Glas Espinel, durante la inauguración del programa Progresar, que impulsa la Corporación Financiera Nacional (CFN) y que tiene como objetivo otorgar créditos a pequeños y medianos emprendedores, quienes no disponen de las garantías necesarias que solicitan las instituciones financieras para entregarles líneas de crédito.

El Vicepresidente afirmó que es necesario arriesgar. "Se necesita la decisión de arriesgar, arriesgarse a emprender, a construir e innovar. Ese es el gran desafío de todos los ecuatorianos", señaló Glas, quien enfatizó que ya el país tiene las condiciones para despegar en el emprendimiento e innovación.

El programa Progresar, que nació luego de que el Fondo de Garantías para la Micro y Pequeña Empresa (Fogamype) superara las expectativas, es una de las primeras medidas concretas para el cambio de la Matriz Productiva y significa también un hito en el sistema financiero nacional"

Es importante apoyar a los micro emprendimientos puesto que si estos se llegan a desarrollar positivamente, beneficia de muchas formas ya sea que genera fintes de empleo y con esto ayuda a disminuir la tasa de desempleo del país, y por otro lado ayuda a generar ingresos al país. Para los pequeños y medianos emprendedores uno de los limitantes para llevar a cabo su idea ha sido la dificultad de acceder a un crédito, es por esto que el Vicepresidente Jorge Glass, se ha dedicado en los últimos tiempos a apoyar a los micro emprendimientos en conjunto con la CFN.

\section{Resultados.}

Posteriormente como resultado, se coloca a disposición la información de nuestra investigación.

Todos sabemos que los micro-emprendimientos familiares son una pauta para alcanzar grandes logros empresariales, también son una parte importante en el mundo empresarial 
y en la economía del país, porque ayuda a solventar las necesidades básicas de la sociedad en general, a aceptar desafíos y a responder a las exigencias cambiantes de los consumidores.

Una de las características más relevantes de los micro-emprendimientos, comparados con las grandes empresas es que son más dinámicas, flexibles (habilidad para cambiar rápidamente de dirección), adaptables y son capaces de responder a las condiciones cambiantes del entorno.

Según Ecuador Cifras (2014), el índice de Actividad Emprendedora Temprana, conocido como TEA, es del 15,82\% en Ecuador. Esto quiere decir que siete de cada 10 ecuatorianos adultos están en proceso de iniciar un negocio o gestionando una nueva empresa (de no más de 42 meses). Pese al dinamismo que evidencian los números, este índice -considerado el eje del GEMha registrado un importante descenso respecto del 2004, la primera vez que se aplicó el estudio localmente, cuando el TEA fue del 27,2\%, y ubicó al Ecuador en el tercer lugar del ranking mundial.

En el Ecuador se conoce que el $90 \%$ son micro-emprendimientos familiares, se puede decir que cada día se va creando una, pero solo son algunas las que sobreviven en el transcurso, o a su vez duran poco y los cierran, estos emprendimientos son capaces de transformar al mundo por su innovación, su forma de trabajar en equipo y ofrecer sus productos. Por todo eso los micro-emprendimientos familiares han llegado a alcanzar una cima alta a nivel empresarial, varios de ellos se han convertido en un éxito total que han sido reconocidas a nivel nacional y también internacional, no ha sido una tarea fácil pero la unión y la fuerza de todos los miembros de la familia denota que todas las barreras se pueden superar.

Los microemprendimientos familiares en la industria del calzado en Tungurahua existen una gran proporción de fábricas y talleres textiles en los sectores de Cevallos, Quero, Mocha y Tisaleo.

Un dato importante que debemos destacar es que la industria y confección de calzado a nivel nacional en el último año genero alrededor de 465 millones de dólares cifras importantes para el PIB.

Más de la mitad de los emprendedores ecuatorianos están entre los 25 y 44 años de edad y la incorporación de mujeres a este segmento es cada vez más significativa. La diferencia radica en que los hombres emprenden primordialmente por oportunidad, mientras que las mujeres son más sensibles a la necesidad. Esta motivación usualmente es más determinante en segmentos que no han tenido la oportunidad de acceder a educación formal o que han completado un nivel medio.

Ecuador consta entre los 15 países con mayor tendencia a iniciar nuevos negocios. Las fortalezas de sus emprendedores son la juventud y la capacidad de detectar oportunidades del mercado; sus debilidades, la falta de capital y la poca capacitación. El emprendimiento puede ser un motor de la economía, pero su impacto aún es incipiente. 


\section{Resumen.}

SEGÚN (ACEVEDO, 2009), (ALLES, 2005), (CRUZ VARGAS, 2001), (LESANSKI, 2001) (VIVANCO, 2011), (tORCIGLIANI, 2007).- Micro emprendimiento son los diferentes tipos de empresas pequeñas que generan ganancia, las cuales son propiedad y administrados por los propios emprendedores, los cuales son los propios operarios de estas pequeñas organizaciones, y que generalmente no cuentas con gran cantidad de empleados. Se trata de emprendimientos individuales o familiares que requieren una baja inversión. Su forma jurídica puede llamarse economía informal. También se dice que es un desafío que tienen los sectores más pobres de un país para superar la pobreza y poder sustentar los gastos de la familia. Son organizaciones que no cuentan con más de diez empleados, las cuales son una opción frente al subempleo y a la desocupación de las personas. Estas pequeñas empresas ofrecen una gran variedad de actividades productivas y de servicios. Para su elaboración o empezar un proyecto de estos no se necesita mucho capital y bajos costos operativos.

SEGÚN (Blaser, 2003) (Coraggio, 2012) (Ramos, 2013) concuerdan que el micro emprendimiento en general como los familiares tienen ciertas características propias como son:

- Goza de ventajas crediticia otorgada por ciertos bancos con el propósito de fomentar su desarrollo y combatir el desempleo.

- El emprendedor necesita de asesores externos porque no cuenta con los conocimientos requeridos.

- Quien emprende un negocio está dispuesto a arriesgar cierto capital.

- Intenta encontrar presupuestos convenientes para la adquisición de productos materiales.

- Tienen una reducida cantidad de miembros.

- Son dirigidos por los propietarios.

- Su capital no es significativo, la mano de obra es mucho más importante.

- La comunicación es permanente por tratarse de pocas personas.

- Se aceptan los cambios sin mayores dificultades.

- La administración y gerencionamienyo es intuitiva.

- Poseen pocos activos.

- Tiene posibilidad de adaptarse a los cambios rápidamente

\section{Micro emprendimientos Familiares.}

Según (Valenzuela, 2005) (Internos, 2008) (Financiera, 2015)

La microempresa es definida como aquella que considera el rango de 1 a 10 trabajadores y todos o casi todos son miembros de la familia, se toma en cuenta el valor de activos fijos hasta $\$ 20.000$ y ventas hasta $\$ 50.000$. Es aquella que utiliza tecnología simple o 
adaptada, mano de obra intensiva y con un coeficiente menor a \$1000. Su organización utilizada en la microempresa es familiar, incluyendo la actividad económica realizada al interior de la vivienda.

La microempresa familiar es una empresa que pertenece a uno o más personas naturales que realizan actividades empresariales en la casa habitación en que residen. Las labores profesionales, oficios, comercio, pequeñas industrias o talleres, artesanía o cualquier otra actividad lícita que se realizan en ese domicilio, son entonces, de prestación de servicios o de producción de bienes. Sin embargo, la ley excluye aquellas actividades consideradas peligrosas, contaminantes y molestas.

\section{Ventajas e inconvenientes de la microempresa familiar.}

La principal ventaja de una microempresa es la flexibilidad que caracteriza a este tipo de negocios, debido a su estructura simple, que le permite una mejor y rápida adaptación al mercado.

Sin embargo, las microempresas familiares cuentan con algunas desventajas, como la falta de financiación para poder poner en práctica sus planes de expansión, tanto geográfica como tecnológica. Esta falta de financiación normalmente acarrea limitaciones a la hora de competir con otras empresas e impide las posibilidades de crecimiento.

\section{Características:}

- Funciona necesariamente, en la casa habitación familiar.

- Puede pertenecer a una o más personas naturales que residan en casa.

- Los activos productivos de la microempresa no deben superar los 1.000 unidades de fomento (se excluye el valor del inmueble.

- No deben trabajar en ella más de cinco personas extrañas a la familia.

- Cada empresa familiar requiere previamente autorización Sanitaria.

Una empresa en su definición más estricta es una "acción dificultosa que se acomete con resolución". Entonces empresa familiar será aquella "acción dificultosa que una familia comete con resolución" y para ello compromete sus bienes propios (patrimonio) para alcanzar el éxito.

Podemos considerar que una empresa familiar es una unidad productiva, generalmente de tamaño pequeño, que pertenece al núcleo familiar que la fundo y al dirige un miembro de ese núcleo familiar.

\section{Desventajas y problemas en la empresa familiar.}

Las empresas familiares son los ejes de nuestra sociedad. En la mayoría de las empresas, pequeñas, medianas y grandes, están involucrados parientes y amigos sobre quienes descansan grandes responsabilidades. Esto ayuda a generar riqueza, y a unir esfuerzos, pero suelen presentarse diversos problemas: 
1. La sucesión (proceso que se enfrenta tarde o temprano)

2. Centralización del poder (demasiada responsabilidad)

3. Organización

4. Nepotismo y manipulación (Abuso del poder de parientes o amigos)

5. Tensiones en la brecha generacional.

\section{Principales Micro emprendimientos Familiares en el Ecuador}

Según página de Industria del calzado Ecuador (Ecuador, 2014)(página oficial de gerentes de calzado a nivel nacional) menciona que los microemprendimientos nacieron de pequeños talleres familiares, con su ingenio e innovación surgieron al mundo empresarial fomentando el espíritu emprendedor que existe en los ambateños y hoy son reconocidos a nivel nacional y además su producto es enviado a varios países por su calidad y diseño.

En el país cada año se realiza una feria dedicada al calzado donde los fabricantes tanto nacionales como extranjeros exponen sus productos. En dichas ferias el sector de Tungurahua siempre ha sido criticado fructuosamente por la calidad de sus productos, la excelente materia prima y por los precios competitivos.

A continuación se mencionan algunas empresas que nacieron de un microemprendimiento y que hoy en día se encuentran constituidas en el mercado, cuentan con un gran número de clientes y varios nichos de mercado posicionados por estas.

Principales microemprendimientos de calzado en Ambato - Tungurahua.

- Nerverland Footwear Teléf.: 032843707

- Dublauto Imporcalza Teléf.: 032829 - 145 / 032829 - 133

- Marjorie Botas Teléf.: $03285-0667$

- Calzado Zepol Teléf.: $03246-7025$

- Calzado Misshell Bosseli Teléf.: 03243 - 6792

- Creaciones Anabelle Teléf. 03242 - 3946

- Creaciones Vanessa Teléf. 03246 - 0534

- Calzado Luigi Valdini Teléf. 032 854-249 / 032 854-471

- Calzado Deportivo GOB Shoes Teléf. 03245 - 1119

- Calzado Dovian

- Calzado Venus

- DACRIS Shoes Teléf.: 099555 - 4083 / 098342 - 6410

- Calzado Trajano Basantes Teléfono: 03285 - 2672

- Buffalo Calzado Industrial Teléfono: 03241 - 0094 / 032841 - 4 


\section{Informe CALTU.}

La industria del calzado ha experimentado un importante crecimiento desde el 2009. Datos obtenidos de la Cámara de Calado de Tungurahua (Caltu), señalan que de los 15 millones de pares de zapatos que se producían en el 2008, se pasó a 28,8 millones en el 2011 desde ese año ha ido ascendiendo la cifra. Es decir, en el transcurso de estos años, el nivel de facturación se incrementó en un 15\% según el Ministerio de Industrias.

Una de las principales razones para este repunte es la aplicación del arancel mixto, que entro en vigencia desde el 01 de junio del 2010.

Con estos aranceles, que imponen un gravamen del 10\% a cada para importado, se trata de proteger al calzado nacional, principalmente ante a los productos con costos más reducidos que ingresaban de China, Colombia o Perú.

En el año 2008, en el país existía alrededor de 600 empresas productoras de calzado que nacieron como un microemprendimiento que estaban a punto de quebrar, pero gracias a las medidas gubernamentales se dinamizo este sector.

Según datos del Censo Económico, realizado por el Instituto Nacional de Estadística y Censos (INEC), en el país, existen 870 establecimientos que se dedican a la producción de calzado.

Mientras que CALTU indica que hasta este año están registrados 4500 productores, a escala nacional, entre grandes, medianos y artesanos. De esta cantidad, el 50\% pertenecen a la provincia de Tungurahua.

Con zapatos nacionales a precios competitivos, se produjo el efecto domino en el mercado, que desencadeno en mayores compras. Cifras del Ministerio de Industrias y Productividad (MIPRO) muestran que, en el 2010, la venta interna de calzado nacional represento USD 165,7 millones y para el siguiente año escalo a 318 millones.

CALTU también ayuda a que muchas firmas tungurahuenses lleguen a varios locales comerciales de Guayaquil, Cuenca, Loja, Quito, Manta y Machala. Los zapatos se venden en cadenas comerciales como Etafashion, DePrati y Casa Tosi.

Actualmente una de las empresas que envía mercadería al exterior es Plasticaucho Industrial S.A, esta compañía, también ambateña, exporta zapatos de lona, botas de caucho y calzado escolar de cuero a Colombia y Perú.

\section{Conclusiones.}

- Los micro emprendimientos de calzado en el Ecuador en la provincia de Tungurahua se han ido desarrollando cuando la gente del sector vio la necesidad 
de salir de la pobreza y es ahí cuando empezaron los pequeños proyectos de calzado ya que gracias a que existe varios proveedores de cuero se hace fácil la adquisición de la materia prima.

- De los pequeños proyectos que surgen en la provincia de Tungurahua en la ciudad de Ambato han ido creciendo poco a poco han llegado a posicionarse de una gran manera competitiva en el mercado como un gran ejemplo tenemos la empresa Gamos que gracias a la perseverancia que han tenido sus dueños y trabajadores se convirtió en una de las empresas principales de de calzado en Ambato.

- El micro emprendimiento se han convertido para la sociedad general en un reto porque después de idear la manera de salir adelante con pequeños talleres que son integrados por los propios miembros de la familia como recurso humano y con una pequeña cantidad como capital, estas personas han hecho de su negocio grandes empresas que en la actualidad son reconocidas a nivel nacional o en otros casos han llegado a internacionalizarse, es así como en este caso la provincia de Tungurahua en el área de calzado tiene a varios autores de firmas reconocidas.

- Para el PIB el micro emprendimiento familiar en la industria del calzado en los últimos años han representado cifras importantes. En el Ecuador el $90 \%$ son emprendimientos familiares, es decir que cada día existe alguien con una idea de crear algo nuevo y demostrar que en el Ecuador existe gente con pensamiento innovador.

\section{Bibliografía.}

ACEVEDO, D. (2009). PRIMER FORO DE JOVENES DEL MECOSUR. CHILE: BIANCHI.

\section{ALLES, M. (2005). AUTOEMPLEO. BUENOS AIRES: GRANICA.}

Blaser, M. (2003). Investigacion Evaluativa de FOPOSOL. San Carlos.

Coraggio, J. L. (5 de Occtubre de 2012). Economia de los sectores populares. Bogota.

CRUZ VARGAS, A. (2001). EXPERINCIAS Y PERSPECTIVAS DEL PROYECTO DE GENERACION DE EMPLEO RURAL. BOGOTA: TICA.

Ecuador, C. (2014). Directorio de calzado en Ecuador . Obtenido de http://www.calzadoecuador.com/

Financiera, N. (Febrero de 2015). La pequeña empresa familiar . Obtenido de Instituto PYME: http://mexico.smetoolkit.org/mexico/es/content/es/3528/Lapeque $\%$ C3\%B1a-empresa-Familiar

Ing. Carlos Morlás Espinoza . (2006 ). Ecuador ¿Pais de Emprendedores? Guayaquil: Ecotec. 
Internos, S. d. (marzo de 2008). Microempresas Familiares . Obtenido de http://www.sii.cl/contribuyentes/empresas_por_tamano/microemp_familiares.ht $\mathrm{m}$

LESANSKI, P. (2001). UNA EMPRESA EN MARCHA. BAHIA BLANCA: SAN VAL, INCORPORATED.

Ramos, E. S. (2013). Estudio CEDES . Espana.

Ruiz, A. G. (2006). Empresas Familiares en Ecuador: El caso del Grupo Godoy . ESPAE Y EMPRESA , 11 - 13.

tORCIGLIANI, I. (2007). MICROEMPRENDIMIENTOS FAMILIARES: INTERVENCION DEL TRABAJO SOCIAL. ESPAÑA: ESPACIO EDITORIAL.

Valenzuela, M. E. (2005). ¿Nuevo sendero para las mujeres? Microemprendimientos en America Latina. Buenos Aires : LOM ediciones / CEM .

VIVANCO, V. (2011). MAMAT, EMPRENDIMIENTOS PARA MAMAS. CHILE: PONTIFICIA.

www.buenvivir.gob.ec/

\section{Para citar el artículo indexado.}

Vargas A., Reinoso G. \& Pico I. (2017). Micro Emprendimientos Familiares de Calzado en Tungurahua. Revista electrónica Visionario Digital 1(1), 5-18. Recuperado desde: http://cienciadigital.org/revistacienciadigital2/index.php/VisionarioDigital/article/view/ $\underline{239 / 531}$

\section{Ciencia LDigital}

El artículo que se publica es de exclusiva responsabilidad de los autores y no necesariamente reflejan el pensamiento de la Revista Ciencia Digital.

El articulo queda en propiedad de la revista y, por tanto, su publicación parcial y/o total en otro medio tiene que ser autorizado por el director de la Revista Ciencia Digital
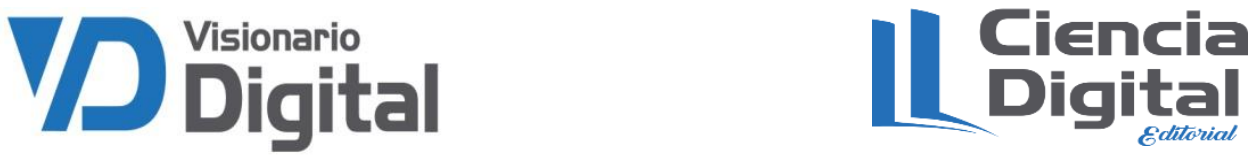